\title{
Catalytic Reactions of Living Polymers: Density Functional Study of Reactivity of Phenol and Phenoxides with the Cyclic Tetramer of Polycarbonate
}

\author{
P. Ballone,$\stackrel{\dagger}{\dagger}$ B. Montanari,, and R. O. Jones* \\ Institut für Festkörperforschung, Forschungszentrum Jülich, D-52425 Jülich, Germany
}

Received: October 19, 1999; In Final Form: January 5, 2000

\begin{abstract}
The reactivity of phenol, lithium phenoxide $(\mathrm{LiOPh})$, and sodium phenoxide $(\mathrm{NaOPh})$ with the cyclic tetramer of bisphenol A polycarbonate (BPA-PC) has been investigated using density functional calculations. The potential energy of the system is computed using a suitable reaction coordinate and relaxing all other degrees of freedom by $\mathrm{Car}-$ Parrinello molecular dynamics. Both $\mathrm{LiOPh}$ and $\mathrm{NaOPh}$ catalyze ring opening with small energy barriers ( $\Delta E=4.0,2.5 \mathrm{kcal} / \mathrm{mol}$, respectively) to a chain with a phenyl carbonate at one end and a phenoxide at the other, a "living polymer". The barrier is large for phenol $(\Delta E>40 \mathrm{kcal} / \mathrm{mol})$, but the total energy differences between the reactants and the chain are very small in all three molecules. We discuss the balance between changes in entropy and energy, and we compute the vibrational properties of metastable intermediate species.
\end{abstract}

\section{Introduction}

The wide range of industrial applications of polycarbonates has led to many studies, and bisphenol A polycarbonate (BPAPC) has been a prototype for both theorists and experimentalists. ${ }^{1}$ Information about the geometric arrangement of the atoms is essential for discussing the properties of any material. The most detailed has been provided in the polycarbonate context by X-ray diffraction studies of molecules closely related to BPAPC, examples being the crystalline forms of DPBC $\left[4,4^{\prime}\right.$ -isopropylidenediphenylbis(phenyl carbonate)]. ${ }^{2}$ Furthermore, the cyclic dimer, trimer, and tetramer carbonates of BPA have been isolated and analyzed spectroscopically, ${ }^{3,4}$ and the structures of the crystalline forms of dimer and tetramer determined by X-ray diffraction. ${ }^{4}$ The cyclic tetramer of BPA-PC (Figure $1)^{3,4}$ provides an ideal model for theoretical studies of the microscopic properties of polycarbonate. It has a very large, open cyclic structure, with a cavity of $14-16 \AA$ and outside diameter of $20.7 \AA,{ }^{4}$ and the size of the ring (132 atoms) means that there is no hindrance to ring-flipping or cis-trans-transtrans isomerization about the carbonyl group. ${ }^{4}$ The tetramer should then reproduce much of the local bonding behavior found in a single polymer chain, and cyclic oligomers in general are useful intermediates for the preparation of high molecular weight polycarbonate. ${ }^{5}$ Ring-opening polymerization, $\mathrm{ROP},{ }^{6}$ is often initiated by nucleophilic attack, giving rise to a "living" polymer. $^{7}$

The present work describes density functional calculations of the reaction of the cyclic tetramer with the molecules phenol, lithium phenoxide $(\mathrm{LiOPh})$, and sodium phenoxide $(\mathrm{NaOPh})$. $\mathrm{NaOPh}$ has often been used as a reactant, one of the best known examples being its carboxylation to salicylic acid (KolbeSchmitt reaction), ${ }^{8}$ and it initiates polymerization in cyclic carbonates. ${ }^{6}$ The crystallization of PC is increased by several orders of magnitude by the presence of alkali metal aryl

\footnotetext{
* Corresponding author (e-mail: r.jones@fz-juelich.de).

$\dagger$ Permanent address: Università degli Studi di Messina, Dipartimento di Fisica, Contrada Papardo, I-98166 Messina, Italy.

$¥$ Present address: Department of Physics, Queen’s University, Belfast BT7 1NN, Northern Ireland.
}

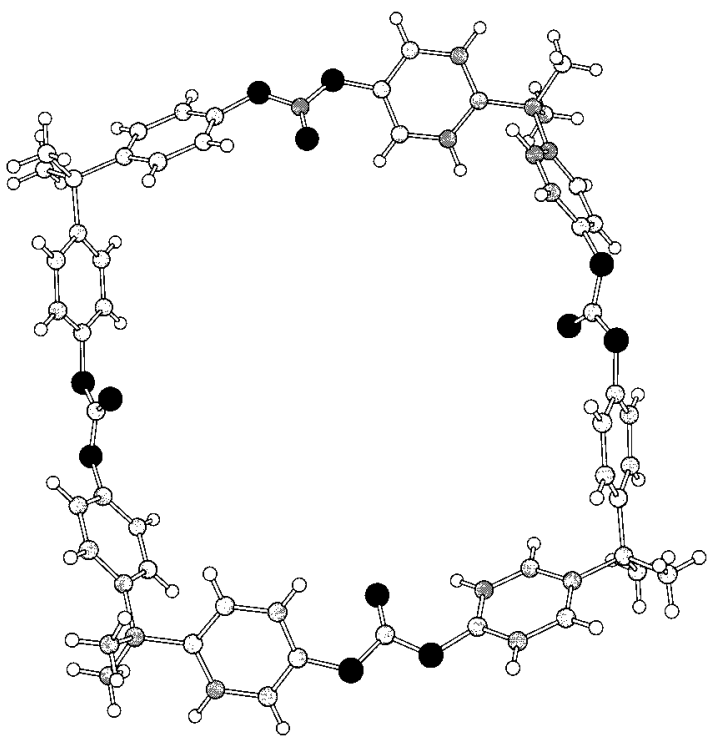

Figure 1. Cyclic tetramer of BPA-PC: $\mathrm{C}$ atoms, gray; $\mathrm{O}$, black; $\mathrm{H}$, white.

carbonates such as sodium benzoate, and this has been attributed to the formation of $\mathrm{NaOPh}$ chain ends that catalyze a fast transesterification reaction. ${ }^{9}$ The polymerization of macrocyclic oligomers of BPA-PC has been initiated by numerous ions, and the reaction of $\mathrm{LiOPh}$ with the cyclic tetramer is unusual, since it has no measurable exotherm (enthalpy less than $0.3 \mathrm{kcal} /$ $\mathrm{mol})^{10}$ and must be entropy driven. Experimental studies on a number of functionalized bisphenols using a variety of catalysts showed that the carbonate group provided the site for ringopening polymerization in all cases. ${ }^{11}$

These calculations continue a series of applications of the density functional (DF) method $^{12}$ to organic molecules, molecular crystals, and polymers, including the cyclic tetramer. ${ }^{13}$ The DF calculations are free of adjustable parameters and, when combined with molecular dynamics (MD), ${ }^{14}$ allow us to simulate the microscopic dynamical behavior of systems and to avoid geometrical structures corresponding to unfavorable local 
minima in the energy surface. Our earlier work ${ }^{2,15}$ has showed that DF calculations-with the exchange-correlation energy functional proposed by Perdew, Burke, and Ernzerhof ${ }^{16}$ - provide a satisfactory description of the intermolecular interactions in crystalline forms of polyethylene (PE) and DPBC. Rotation barriers in small organic molecules are described well by both local density and gradient-corrected functionals, ${ }^{17}$ although both tend to underestimate reaction barriers. ${ }^{18}$ Nevertheless, DF calculations provide the only viable method at present to identify reaction mechanisms in systems as large and as complex as those studied here, to account for the atomic relaxations during the reaction, and to provide estimates of the energy barriers. Moreover, the structural rearrangements involved are relatively minor, and only the intermediate stages display species with a different chemical configuration. Essential details of the calculations are provided in section II. The properties of the isolated phenol, $\mathrm{LiOPh}$, and $\mathrm{NaOPh}$ molecules are discussed in section III, and the reactions of these three molecules with the cyclic tetramer are described in section IV. The vibration frequencies found for various structural units are presented in section $\mathrm{V}$. Our findings are summarized in section VI, where we also discuss the balance between energy and entropy during the reactions.

\section{Method of Calculation}

The method of calculation was described in detail previously. ${ }^{19,20}$ The electron-ion interaction is represented by ionic pseudopotentials with the (nonlocal) form suggested by Troullier and Martins. ${ }^{21}$ We adopt a plane wave basis with a kinetic energy cutoff of $35 \mathrm{au}$, and the expansion of the orbitals uses a single point $(\mathrm{k}=0)$ in the Brillouin zone. Periodic boundary conditions are assumed, and we use an orthorhombic cell with lattice constants 58.6, 41.0, and $26.5 \AA$. Density functional calculations presently require an approximation to the exchangecorrelation energy. Our previous calculations on organic molecular crystals ${ }^{15}$ indicated that the PBE approximation, which is relatively simple and free of empirical input, is suitable in this context. It is used in the present work. All structures for the reactants and products were optimized using a combination of DF calculations with $\mathrm{MD},{ }^{22}$ and the coordinates of all atoms are allowed to relax. The vibration frequencies and eigenvectors of the molecules were calculated for the optimized geometries discussed in section V. The dynamical matrix $\mathbf{D}$ is evaluated using finite differences with atomic displacements $\delta$ of the order of $0.005 \AA$. The computed frequencies change by at most a few $\mathrm{cm}^{-1}$ upon increasing or decreasing $\delta$ by a factor of 3 , so that anharmonic contributions are very small. The intensity of infrared fundamentals in absorption $\alpha$ for a mode with frequency $v_{j}$ is estimated by associating an electrostatic charge $q_{I}$ with each atom $I$ and evaluating

$$
\alpha\left(v_{j}\right)=\frac{8 \pi^{3} N v_{j}}{3 c h}\left|\sum_{I} q_{I} \delta \mathbf{r}_{I}^{(j)}\right|^{2}
$$

where $\delta \mathbf{r}_{I}(j)$ is the displacement of atom $I$ for the $j$ th vibrational eigenvector and $N$ is the molecular density. The atomic charges $q_{I}$ are computed by a least-squares fit to the electrostatic potential around each molecule. ${ }^{23}$

\section{Isolated Phenol, LiOPh, and NaOPh Molecules}

The reactions studied here are initiated by the molecules shown in Figure 2: (a) phenol, (b) $\mathrm{LiOPh}$, and (c) $\mathrm{NaOPh}$. Some properties of the isolated molecules that could influence their
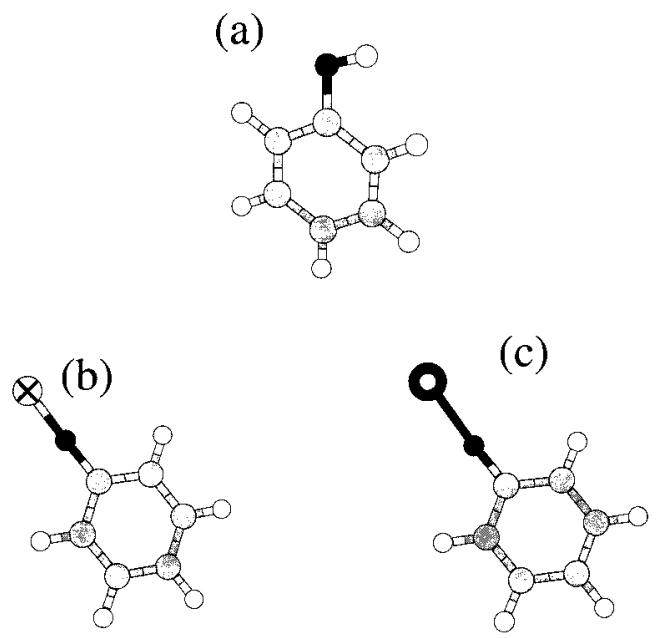

Figure 2. Molecules studied in the present work: (a) $\mathrm{LiOPh}$; (b) $\mathrm{NaOPh}$; (c) phenol. Key: $\mathrm{C}$ atoms, gray; O, black; $\mathrm{H}$, white; Li, crosses; $\mathrm{Na}$, double circles.

TABLE 1: Calculated Properties for Molecules $\mathrm{C}_{6} \mathrm{H}_{5} \mathrm{OM}(\mathrm{M}$ $=\mathbf{H}, \mathbf{L i}, \mathbf{N a})^{a}$

\begin{tabular}{llll}
\hline & $\mathrm{M}=\mathrm{H}$ & $\mathrm{M}=\mathrm{Li}$ & $\mathrm{M}=\mathrm{Na}$ \\
\hline dipole moment $(\mathrm{D})$ & 1.3 & 7.6 & 12.0 \\
$q(\mathrm{M})(\mathrm{e})$ & +0.35 & +0.83 & +0.91 \\
$q(\mathrm{O})(\mathrm{e})$ & -0.53 & -1.10 & -1.11 \\
$q(\mathrm{C})(\mathrm{e})$ & +0.40 & +0.82 & +0.84 \\
$d(\mathrm{M}-\mathrm{O})(\AA)$ & 0.98 & 1.56 & 2.11 \\
$\alpha(\mathrm{C}-\mathrm{O}-\mathrm{M})(\mathrm{deg})$ & 108 & 180 & 180 \\
$\Delta E\left(\mathrm{H}_{2} \mathrm{O}\right)(\mathrm{kcal} / \mathrm{mol})$ & 6.6 & 16.5 & 10.3
\end{tabular}

${ }^{a} \Delta E\left(\mathrm{H}_{2} \mathrm{O}\right)$ is the cohesive energy of the complex with a water molecule bound to the terminal atom $\mathrm{M}$.

interaction with polycarbonate chains, particularly the structure and charge distribution, are given in Table $1 . \mathrm{LiOPh}$ and $\mathrm{NaOPh}$ are rather similar, each having a linear $-\mathrm{C}-\mathrm{O}-\mathrm{M}(\mathrm{M}=\mathrm{Li}$, $\mathrm{Na}$ ) termination and an $\mathrm{O}-\mathrm{M}$ bond that is predominantly ionic (as revealed by the analysis of the atomic charges and the Mayer bond order ${ }^{24}$ ). The dipole moments are large and oriented along the linear termination. In phenol, by contrast, the $\mathrm{O}-\mathrm{H}$ termination has a strong covalent component (the stretching frequency of $3530 \mathrm{~cm}^{-1}$ is typical of the hydroxyl group), the $-\mathrm{C}-\mathrm{O}-\mathrm{H}$ angle is bent, both the $\mathrm{C}$ and $\mathrm{O}$ atoms have a small electrostatic charge, and the dipole moment is much less. As a measure of the ability of these molecules to bind to closedshell molecules, we have computed their binding energy with a single water molecule. The bond where the oxygen in $\mathrm{H}_{2} \mathrm{O}$ is the acceptor for the $-\mathrm{M}$ termination of phenoxide is strongest in $\mathrm{LiOPh}$. The binding energy of the hydrogen bond in phenol $(6.6 \mathrm{kcal} / \mathrm{mol})$ is in good agreement with previous theoretical estimates. ${ }^{25}$ The present calculations do not allow us to make an unambiguous separation into covalent and ionic components. Nevertheless, all these bonds (particularly water-LiOPh) have a covalent component, since the trends cannot be accounted for by the computed atomic charges and standard values of the ionic radii.

\section{Reactions with Phenol and Phenoxides}

Computer simulations cannot sample all possible reaction paths, and the most favorable found may depend on the initial geometry, the number of points sampled on the path, and the accuracy of the local relaxation procedure. Such effects have been reduced by performing two simulations on each system, with the initiating molecule outside and inside the tetramer ring, respectively, and not in its average plane. The initial reaction 
SCHEME 1: (a) Reactants and (b) Products of RingOpening of the Cyclic Tetramer by LiOPh

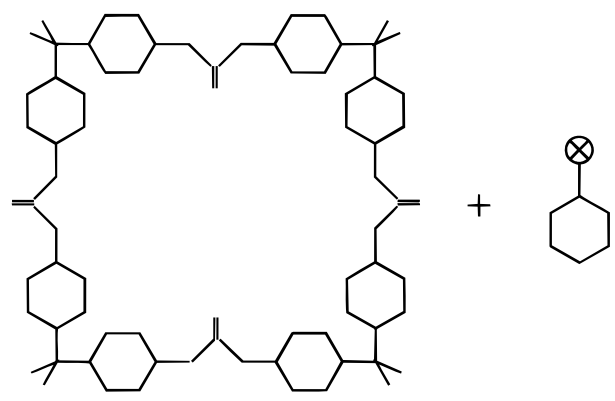

(a)

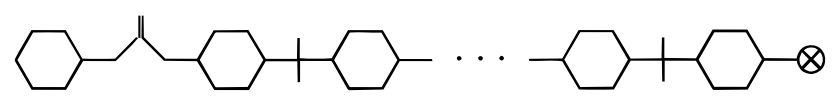

coordinate is the distance $R_{\mathrm{C}}$ between the $\mathrm{C}$ atom of a carbonate group of the ring and the $\mathrm{O}$ atom on phenol or phenoxide. $R_{\mathrm{C}}$ is reduced from values $>5 \AA$, where the interaction is very weak, in progressively shorter steps $(\sim 0.5,0.4$, or $0.2 \AA)$ until a structural transformation or a large energy increase occurs. For each value of $R_{\mathrm{C}}$, we relax all other degrees of freedom of the structure until the energy gradients along the unconstrained directions are less than $10^{-5} \mathrm{au}$. There are qualitative differences between the results for phenol and those for $\mathrm{LiOPh}$ and $\mathrm{NaOPh}$, and we provide full details below. The total potential energy $E_{\text {tot }}$ in phenol increases rapidly as $R_{\mathrm{C}}$ is decreased. We have discontinued the simulation when $\Delta E_{\text {tot }}$ reached $40 \mathrm{kcal} / \mathrm{mol}$, without any significant chemical rearrangement occurring. On the other hand, a reaction takes place in both phenoxides that involves small energy barriers and a nontrivial sequence of characteristic steps. The first stage of all reactions is the formation of a bridge between $\mathrm{M}$ and the $\mathrm{O}$ of the carbonyl group. The reduction in $E_{\text {tot }}$ favors a further approach of the reactant molecule to the cyclic tetramer and allows significant changes in the geometry (particularly in the torsion and bending angles) that allow the reaction to proceed. The change from $\mathrm{sp}^{2}$ to $\mathrm{sp}^{3}$ of the electronic configuration of the carbonate $\mathrm{C}$ atom is crucial, as the compact surroundings of negatively charged $\mathrm{O}$ atoms facilitate the passage of the alkali metal ion around the reaction center. The final state is an open structure with a weak bond between the metal and the new carbonyl group. Thermal effects and the interaction with other carbonate molecules should break this bond readily above room temperature, leading to the final configuration shown in Scheme 1b.

4.1. Lithium Phenoxide. Figure 3 shows six snapshots of the trajectory for the reaction of $\mathrm{LiOPh}$ initially outside the cyclic tetramer, and the corresponding energy changes as a function of $R_{\mathrm{C}}$ are shown in Figure $4 \mathrm{a}$. The two molecules interact very weakly for $R_{\mathrm{C}} \sim 4 \AA$, with minor atomic relaxations and energy changes of less than $1 \mathrm{kcal} / \mathrm{mol}$, and steric forces give rise to an energy barrier of $4 \mathrm{kcal} / \mathrm{mol}$ at $R_{\mathrm{C}}$ $=3.8 \AA$. For shorter separations the $\mathrm{Li}-\mathrm{O}$ attraction produces a rotation of the carbonyl group, the formation of a bond similar to that in the $\mathrm{LiOPh}-$ water complex, and a rapid decrease $(\sim 15$ $\mathrm{kcal} / \mathrm{mol})$ in $E_{\text {tot }}$. The energy stays below the initial value as $R_{\mathrm{C}}$ is reduced to $\sim 1.8 \AA$, where the $\mathrm{C}$ of the original carbonate is surrounded by four $\mathrm{O}$ atoms with approximately tetrahedral coordination $\left(C_{\mathrm{T}}\right)$.

The formation of this configuration (3(3)) is accompanied by an increase in all $C_{\mathrm{T}}-\mathrm{O}$ distances, since the electrophilic $\mathrm{Li}$ atom is shared between the original $\mathrm{O}$ of $\mathrm{LiOPh}$ and the $\mathrm{O}$ atom formerly in the carbonyl group. $C_{\mathrm{T}}$ has a large positive
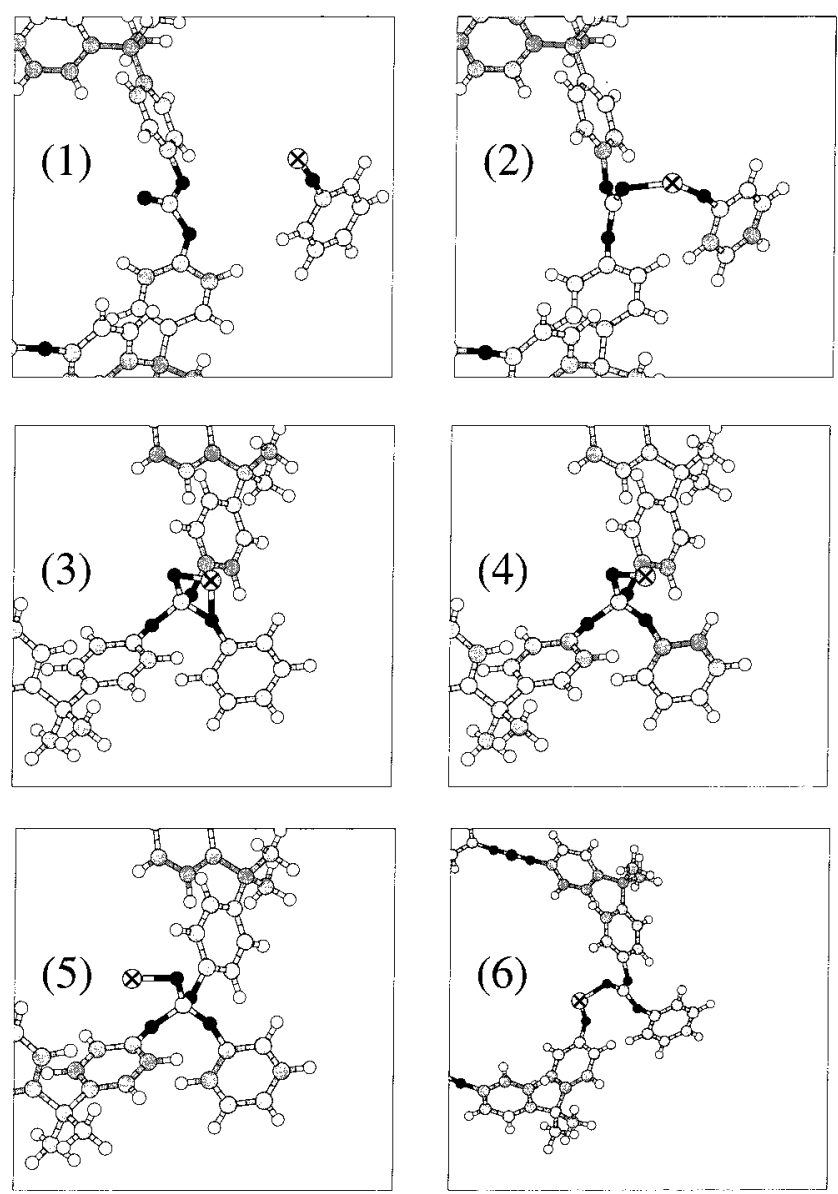

Figure 3. Reaction of $\mathrm{LiOPh}$ with cyclic tetramer of BPA-PC. Only part of the ring is shown.

charge, while the four $\mathrm{O}$ atoms are nearly equivalent, with charges similar to those of other tetrahedral $\mathrm{O}$ atoms in the chain. A reduction of $R_{\mathrm{C}}$ to $1.5 \AA$ (corresponding to a second energy barrier of $\sim 4 \mathrm{kcal} / \mathrm{mol}$ ) is required to symmetrize the tetrahedral geometry of $C_{\mathrm{T}}$ and to break the symmetric bonding of $\mathrm{Li}$. The $\mathrm{Li}$ is now attached to the tetrahedral $\mathrm{O}$ that is bound to a single $\mathrm{C}$ atom (3(4)). The $\mathrm{Li}-\mathrm{O}$ bond is oriented mainly along one of the other three $C_{\mathrm{T}}-\mathrm{O}$ bonds, probably because of the electrostatic attraction between the positive $\mathrm{Li}$ and the electron charge in the covalent bonds. Nevertheless, the $C_{\mathrm{T}}-\mathrm{O}$ bond provides a 3-fold axis for rotations of the $\mathrm{Li}-\mathrm{O}$ bond that change only the $\mathrm{O}-C_{\mathrm{T}}-\mathrm{O}-\mathrm{Li}$ torsion angle and give rise to variations of $E_{\text {tot }}$ of at most $\sim 1 \mathrm{kcal} / \mathrm{mol}$. Under normal thermodynamic conditions, the $\mathrm{O}-\mathrm{Li}$ bond can then be oriented with equal probability along the three directions specified by the $C_{\mathrm{T}}-\mathrm{O}$ bonds at the base of the tetrahedron.

Relaxation of the two equivalent structures with the $\mathrm{Li}-\mathrm{O}$ bond oriented along a $C_{\mathrm{T}}-\mathrm{O}$ bond different from the original one gives rise to structure $\sim(6)$, which has a weak bond between $\mathrm{Li}$ and the new carbonyl $\mathrm{O}$. This geometry is locally stable, with an energy $? 6 \mathrm{kcal} / \mathrm{mol}$ lower than the combined energy of the reactants. The difference in energies between this metastable geometry and 3(2), which is an additional $6 \mathrm{kcal} / \mathrm{mol}$ more stable and has a cis-cis configuration, arises from the less favorable cis-trans configuration of the carbonate group in 3(6). At room temperature, the weak bond between $\mathrm{Li}$ and $\mathrm{O}$ should break easily, leading to the open chain with an active $-\mathrm{C}-\mathrm{O}-\mathrm{Li}$ termination.

The approach of $\mathrm{LiOPh}$ from inside the tetramer ring yields the same product, but the details of the reaction mechanism 


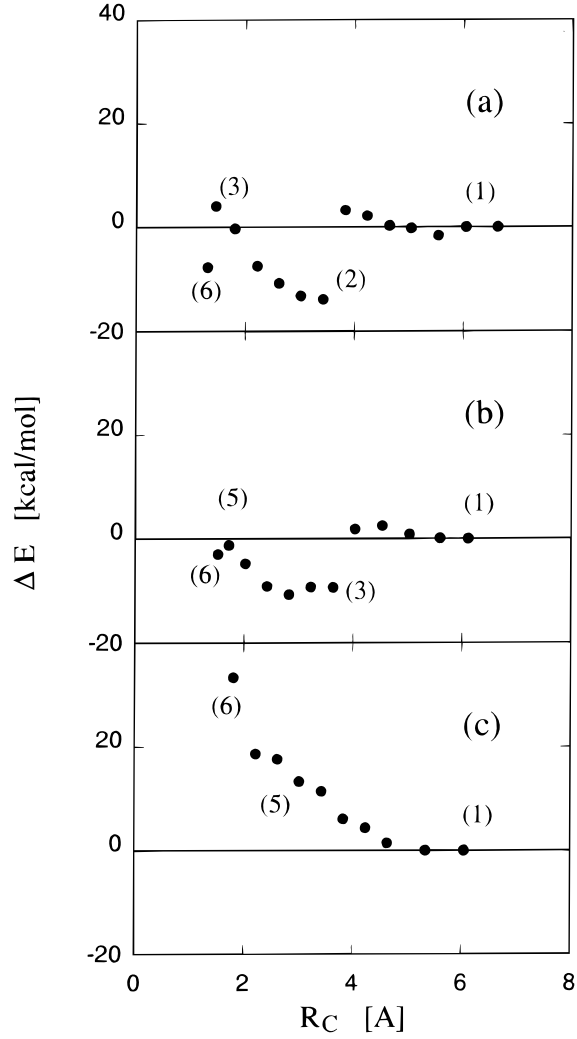

Figure 4. Variation of total potential energy with reaction coordinate $R_{\mathrm{C}}$ during reactions of (a) $\mathrm{LiOPh}$, (b) $\mathrm{NaOPh}$, and (c) phenol. $\Delta E=0$ corresponds to the sum of the energies of the isolated molecules. The numbers denote the related structures in Figures 3, 5, and 6, respectively.

differ. The interaction of the molecules is very weak for $R_{\mathrm{C}}>$ $4 \AA$, but the formation of a bond between $\mathrm{Li}$ and the two tetrahedral $\mathrm{O}$ in the carbonate group $\left(R_{\mathrm{C}} \sim 3.8 \AA\right)$ is accompanied by a lowering of $E_{\text {tot }}$ of $\sim 12 \mathrm{kcal} / \mathrm{mol}$, similar to the first attractive well in the other trajectory. The bonds of $\mathrm{Li}$ to the three $\mathrm{O}$ atoms are of unequal strengths down to $R_{\mathrm{C}}=$ $2.1 \AA$, where the carbonate $\mathrm{C}$ acquires a nearly tetrahedral coordination and an $\mathrm{sp}^{3}$ electronic configuration. At this point, the $\mathrm{Li}$ atom is at the center of the base of the tetrahedron surrounding $C_{\mathrm{T}}$. A reduction of $R_{\mathrm{C}}$ to $1.61 \AA$ leads to an increase in $E_{\text {tot }}$ to $6 \mathrm{kcal}$ above the energy of the reactant molecules, and the tetrahedral coordination of $C_{\mathrm{T}}$ becomes deformed. Further reducing $R_{\mathrm{C}}$ causes one of the $\mathrm{O}-C_{\mathrm{T}}$ bonds of the original carbonate group to lengthen, without a further increase in the energy. This leads to a metastable species similar to 3(6). $E_{\text {tot }}$ is again determined by the configuration of the new carbonate group, and the deformed trans-trans configuration results in a higher energy than in 3(6). Many other trajectories are possible for this reaction, but these two examples show that ring-opening can occur without crossing significant energy barriers.

4.2. Sodium Phenoxide. The similarity of $\mathrm{LiOPh}$ and $\mathrm{NaOPh}$ is reflected in their behavior during reaction with the cyclic tetramer. Six snapshots illustrating the first of the two simulations are shown in Figure 5, and the corresponding energy variations are shown in Figure $4 \mathrm{~b}$. The longer $-\mathrm{O}-\mathrm{Na}$ bond makes it easier for $\mathrm{Na}$ to approach the carbonate (we find no energy barrier) and to form (at $R_{\mathrm{C}}=3.6 \AA$ ) a bridge between the incoming molecule and the carbonyl O. $E_{\text {tot }}$ is lowered by $11 \mathrm{kcal} / \mathrm{mol}$, a similar value to that found in LiOPh. The smaller rotation of the carbonyl required to bond with the incoming $\mathrm{Na}$ appears to compensate for the relative weakness of the bond. The energy remains nearly constant, and the structure remains
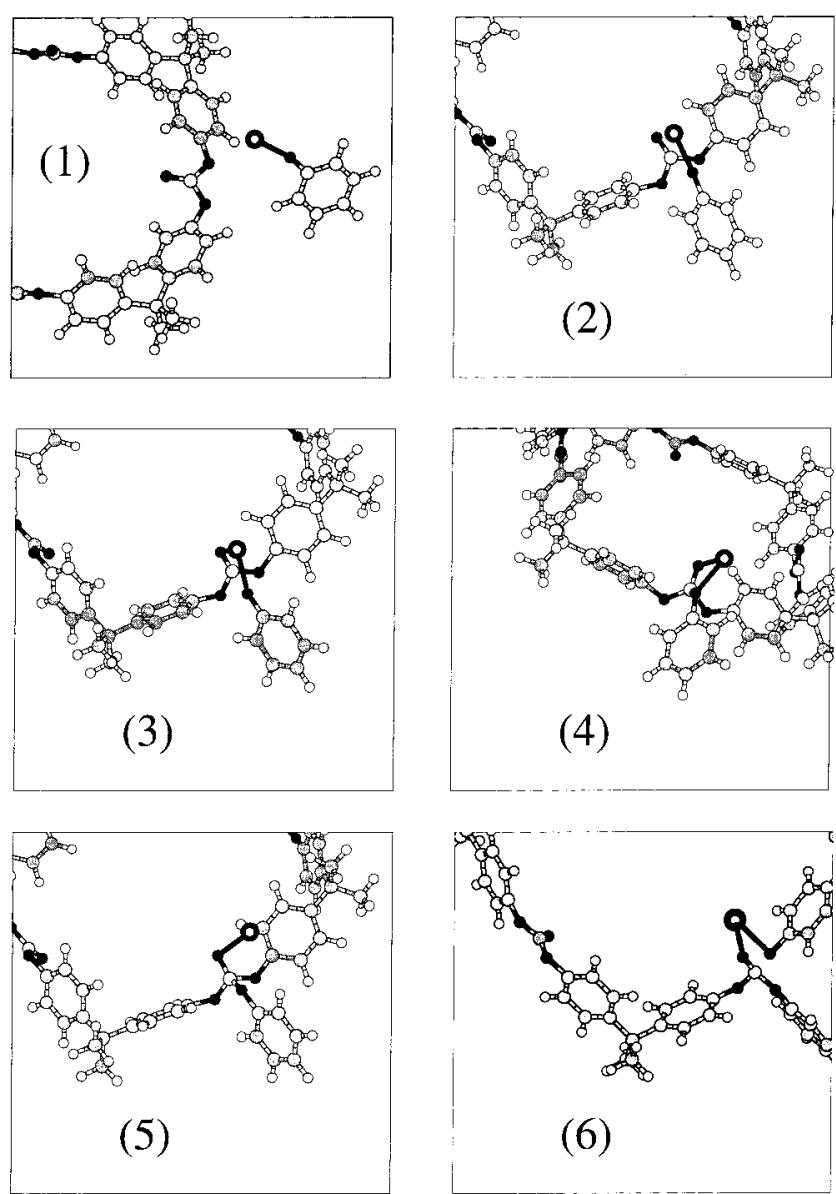

Figure 5. Reaction of $\mathrm{NaOPh}$ with cyclic tetramer of BPA-PC. Only part of the ring is shown.

similar (with $\mathrm{Na}$ shared nearly symmetrically between two $\mathrm{O}$ atoms) on decreasing $R_{\mathrm{C}}$ to $2.01 \AA$. An increase in energy is required to promote the carbonate $\mathrm{C}$ to $\mathrm{sp}^{3}$ coordination, but $E_{\text {tot }}$ remains below the sum of the energies of the isolated reactants. The original $\mathrm{Na}-\mathrm{O}$ bond is broken at $R_{\mathrm{C}}=1.5 \AA$, and $\mathrm{Na}$ remains bound only to the $\mathrm{O}$ previously forming the tip of carbonyl. This bond does not align along one of the other $C_{\mathrm{T}}-\mathrm{O}$ bonds, and the equilibrium value of the $\mathrm{O}-C_{\mathrm{T}}-\mathrm{O}-$ $\mathrm{Na}$ torsion angle changes from 0 to $41^{\circ}$. This difference from the results for $\mathrm{LiOPh}$ appears to be a consequence of the increased size of the metal ion. The related steric hindrance due to the presence of the phenyl rings means that the rotation of the $\mathrm{O}-\mathrm{Na}$ bond around the 3 -fold $C_{\mathrm{T}}-\mathrm{O}$ axis requires more energy $(\sim 4 \mathrm{kcal} / \mathrm{mol})$ than in $\mathrm{LiOPh}$. On the other hand, one of the original $\mathrm{C}-\mathrm{O}$ single bonds opens when the $\mathrm{Na}$ ion approaches it upon a rotation of $120^{\circ}$ from the original direction, as found in $\mathrm{LiOPh}$. Figure 4 shows that $E_{\text {tot }}$ does not lie above its initial value at any stage of the reaction. The trajectory initiating with $\mathrm{NaOPh}$ inside the tetramer ring shows somewhat larger differences from the corresponding simulation for $\mathrm{LiOPh}$, with the longest $\mathrm{Na}-\mathrm{O}$ bond being less favored in forming a bond with the carbonate $\mathrm{O}$ atoms. While $R_{\mathrm{C}}$ decreases to 2.6 $\AA$, the $\mathrm{C}-\mathrm{O}-\mathrm{Na}$ termination of $\mathrm{NaOPh}$ bends by nearly $60^{\circ}$ to maintain the separation of $\mathrm{Na}$ from the (positively charged) central $\mathrm{C}$ atom above $\sim 4 \AA$. A bond between $\mathrm{Na}$ and the two tetrahedral $\mathrm{O}$ atoms of the original carbonate appears to be possible only after this $\mathrm{C}$ atom has tetrahedral coordination at $R_{\mathrm{C}}=2 \AA$. An additional difference between $\mathrm{Li}$ and $\mathrm{Na}$ is that the latter prefers attachment to a single $\mathrm{O}$ atom, although our simulations show that $\mathrm{Na}$ moves from one $\mathrm{O}$ to another unpredictably as $R_{\mathrm{C}}$ changes. Despite these differences, presum- 

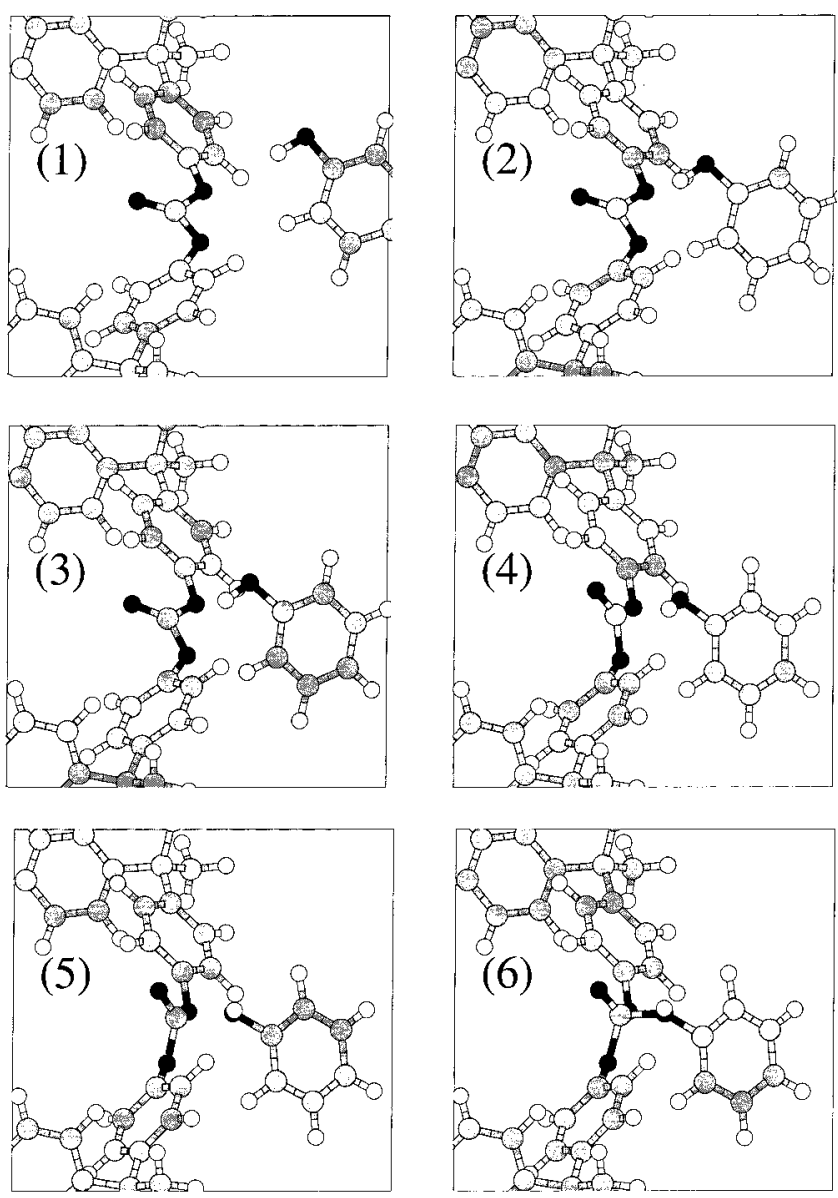

Figure 6. Reaction of phenol with cyclic tetramer of BPA-PC. Only part of the ring is shown.

ably related to the different sizes of $\mathrm{Li}^{+}$and $\mathrm{Na}^{+}$, the structures resulting when $R_{\mathrm{C}}$ is less than $1.5 \AA$ are very similar.

4.3. Phenol. We have noted that the approach of phenol to a carbonate group in the cyclic tetramer results in a purely repulsive potential energy curve, and the energy barrier ( $>40$ $\mathrm{kcal} / \mathrm{mol}$, Figure 4c) is too large for practical reactions. However, the simulation trajectories (Figure 6) show that the reduction of $R_{\mathrm{C}}$ leads to structural changes similar to those found in $\mathrm{LiOPh}$ and $\mathrm{NaOPh}$. The change in $E_{\text {tot }}$ is initially small, the carbonyl group eventually rotates toward the terminal atom of phenol, the central $\mathrm{C}$ atom acquires a tetrahedral coordination, and the nearest phenyl rings rotate to accommodate the incoming molecule. However, we do not observe bond formation between the termination of phenol and the carbonate $\mathrm{O}$ atoms, and there is no potential energy well that allows the molecules to approach and to change the roles of the $C_{\mathrm{T}}-\mathrm{O}$ bonds. Differences in phenol are evident in its structure (the bent $-\mathrm{C}-\mathrm{O}-\mathrm{H}$ bond hinders the approach toward the carbonate $\mathrm{O}$ atoms) and other bonding properties evident in the weak hydrogen bond with water. The same result is also found when the phenol molecule approaches the ring from inside.

\section{Vibrational Properties of Metastable Species}

The reactions of $\mathrm{LiOPh}$ and $\mathrm{NaOPh}$ with the cyclic tetramer take place as a well-defined sequence between transient species whose chemical properties (e.g., the tetrahedral coordination of the carbonyl C) differ from those of both the reactants and product. To aid the identification of these species in future experiments, we have analyzed the vibrations of metastable structures obtained by relaxing structures associated with
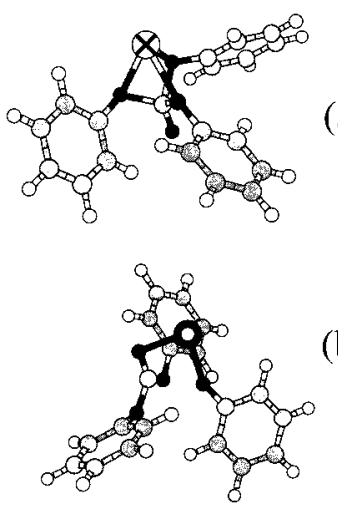

(b)

Figure 7. Metastable structures for which vibration frequencies have been determined: (a) structure observed in reaction of $\mathrm{LiOPh}$ approaching from inside the ring; (b) structure corresponding to 5(3).

shallow potential energy wells along the reaction path. All such structures relax to metastable systems that retain the features of the starting configuration and are stable against dissociation by at least $6 \mathrm{kcal} / \mathrm{mol}$.

To optimize the use of computer resources, we have isolated the active part of the tetramer by (a) cutting the ring beyond the two phenyl rings nearest to the reacting carbonate and (b) saturating the dangling bonds with $\mathrm{H}$ atoms. Our previous computations for a series of carbonate systems ${ }^{13}$ have shown that vibrational and chemical properties are remarkably local and transferable within this family of molecules. Moreover, we describe below a very large computation of the vibrational properties of the entire tetramer that demonstrates this transferability for the metastable species as well. The vibrational properties of two metastable molecules (Figure 7) are determined by the method used in our earlier work on carbonates ${ }^{13}$ and outlined in section VI.

The intermediate species reflect the constraints used in the simulations and differ somewhat from the relaxed structures for which we calculate the vibration frequencies, e.g., the tetrahedral $\mathrm{C}$ atom always relaxes to a highly deformed $\mathrm{sp}^{2}$ configuration. Nevertheless, the significant changes produced by $\mathrm{Li}^{+}$or $\mathrm{Na}^{+}$ in some vibrational modes should aid the identification of the intermediate state. This should be possible if the lifetime of these species is long enough in the reaction environment or by using more initiating molecules or short-time (femtosecond) spectroscopic techniques.

The modes localized on the carbonate group are the most affected by the formation of the complexes, and their ionic character and infrared activity should make them easy to detect. The most obvious differences in all cases arise from the mixing of modes belonging to different symmetries in the isolated molecules. For instance, "in plane" and "out of plane" bending modes for the carbonyl are no longer separated unambiguously, and mixing of bending and stretching modes is observed for the single $\mathrm{C}-\mathrm{O}$ bonds.

In the structure of Figure $7 \mathrm{a}$, the $\mathrm{C}=\mathrm{O}$ stretching mode has a frequency of $1740 \mathrm{~cm}^{-1}$, compared with $1780 \mathrm{~cm}^{-1}$ for the isolated cyclic tetramer. Due in part to mixing with other modes, frequency changes in the $\mathrm{C}=\mathrm{O}$ bending modes are larger, going from the $1139 \mathrm{~cm}^{-1}$ computed for the ring to the $910 \mathrm{~cm}^{-1}$ for the fragment of Figure 7a. Moreover, two new frequencies appear at 533 and $422 \mathrm{~cm}^{-1}$ (stretching and bending of the $\mathrm{O}-\mathrm{Li}$ bonds, respectively) in a spectral region where the isolated tetramer has very low infrared activity. The carbonate structure is less affected by the approaching molecules in Figure 7b, and the stretching mode of $\mathrm{C}=\mathrm{O}\left(1750 \mathrm{~cm}^{-1}\right)$ is closer to the original value. The mode with the strongest $\mathrm{C}=\mathrm{O}$ bending component 
$\left(898 \mathrm{~cm}^{-1}\right)$ results from a mixture of the in-plane $\left(1139 \mathrm{~cm}^{-1}\right)$ and out-of-plane $\left(725 \mathrm{~cm}^{-1}\right)$ modes of the cyclic tetramer. The stretching and bending of the $\mathrm{Na}-\mathrm{O}$ bonds are also mixed, occurring in a frequency range $\left(320-355 \mathrm{~cm}^{-1}\right)$ intermediate between the bending $\left(278 \mathrm{~cm}^{-1}\right)$ and stretching $\left(565 \mathrm{~cm}^{-1}\right)$ of the same bond in $\mathrm{NaOPh}$.

To verify that the results obtained with the fragments are representative of those for larger carbonate molecules, we have repeated the computation for the $\mathrm{Na}$ fragment by considering the entire cyclic tetramer adjacent to $\mathrm{NaOPh}$. The differences in the frequencies of the modes discussed above are small (at most $15 \mathrm{~cm}^{-1}$ ) and show that the use of a truncated molecule is a reliable approximation.

\section{Discussion and Concluding Remarks}

We have performed density functional calculations on the reactions of the cyclic tetramer of BPA-PC and three molecules ( $\mathrm{LiOPh}, \mathrm{NaOPh}$, and phenol). Reactions with BPA-PC are known to take place at the carbonate groups, ${ }^{5}$ and simulations have been performed for each molecule approaching this group from inside and outside the ring. The calculations are performed by choosing an appropriate reaction coordinate and optimizing the positions of all other atomic positions. The total energy of the system and the forces and charges on all atoms are monitored throughout the simulations. The approach of the molecules toward the carbonate group results in initial structural changes that are common to all. The carbonyl $\mathrm{O}$ in the tetramer rotates toward the terminal atom of the approaching molecule, resulting in a change in the coordination of the carbonyl $\mathrm{C}$ from $\mathrm{sp}^{2}$ to $\mathrm{sp}^{3}$. However, the subsequent reaction path and the changes in the total potential energy are very different in phenol, on one hand, and the alkali metal phenoxides, on the other. The energy increases rapidly in phenol, so that a reaction will not take place under normal conditions, while the phenoxides lead to reactions with low energy barriers that result in ring opening. The result is a chain with a phenyl carbonate at one end and a phenoxide at the other (Scheme $1 \mathrm{~b}$ ). The structure and charges of the $-\mathrm{C}-$ $\mathrm{O}-\mathrm{M}$ termination in the phenoxides and in the extended molecules are very similar, so that the "active" termination has been transferred from one molecule to another. Ring-opening may then be viewed as being catalyzed by the phenoxide and giving rise to a "living" polymer.

The driving force toward polymerization can be provided by potential energy or entropy. We have studied this balance by considering the reaction shown in Scheme 1 and computing the total energy of both the separated reactant molecules and the final structure. This reaction does not change the number and type of bonds, and the change in $E_{\text {tot }}$ is very small ( $\Delta E_{\text {tot }}=$ +2 , +1 , and $+1 \mathrm{kcal} / \mathrm{mol}$ for phenol, $\mathrm{LiOPh}$, and $\mathrm{NaOPh}$, respectively). Although the energy differences are negligible in all cases, the energy barriers are much higher in phenol. The calculations indicate that enthalpy cannot be the sole driving force for polymerization in the phenoxides, and the absence of an exotherm agrees with experimental findings. ${ }^{10}$ Entropy changes are likely to play a major role and could be studied further by measuring the temperature dependence of the reaction rate. The computing demands of the DF-based calculations described above rule out their application to long simulations over a range of temperatures, but we are planning an extensive study using a classical force field. ${ }^{26}$ First results on a single ring system (tetramer plus LiOPh) are encouraging. The ring system is very mobile at room temperature and above, and the clear preference for the $\mathrm{LiOPh}$ group to be inside the ring is consistent with the increased number of van der Waals bonds in this geometry. These first calculations indicate that there is a small entropy difference between the systems $a$ and $b$ in Scheme 1. However, the results for a single ring do not represent the interactions in a melt, for example, where the "living" $\mathrm{Li}$ atom can interact with many other molecules. We are extending the classical simulations to cover this case.

Acknowledgment. We thank D. J. Brunelle (GE Corporate Research and Development) for many suggestions and helpful discussions. The DF calculations were performed on the Cray T3E computers in the Forschungszentrum Jülich with generous grants of CPU time from the Forschungszentrum and the John von Neumann Institute for Computing (NIC).

\section{References and Notes}

(1) See, for example: Hutnik, M.; Argon, A. S.; Suter, U. W. Macromolecules 1991, 24, 5970. Tomaselli, M.; Robyr, P.; Meier, B. H.; Grob-Pisano, C.; Ernst, R. R.; Suter, U. W. Macromolecules 1996, 89, 1663. Goetz, J. M.; Wu, J.; Yee, A. F.; Schaefer, J. Macromolecules 1998, 31, 3016 and references therein.

(2) Montanari, B.; Ballone, P.; Jones, R. O. Macromolecules 1998, 31,7784 and references therein.

(3) Schnell, H.; Bottenbruch, L. Makromol. Chem. 1962, 57, 1

(4) Brunelle, D. J.; Garbauskas, M. F. Macromolecules 1993, 26, 2724.

(5) Brunelle, D. J.; Shannon, T. G. Macromolecules 1991, 24, 3035.

(6) Brunelle, D. J. In Ring-Opening Polymerization: Mechanisms, Catalysis, Structure, Utility; Brunelle, D. J., Ed.; Hanser: München, Germany, 1993; p 1, 309.

(7) See, for example: Greer, S. C. Adv. Chem. Phys. 1996, 94, 261

(8) See, for example: Kunert, M.; Dinjus, E.; Nauck, M.; Sieler, J. Chem. Ber./Recueil 1997, 130, 1461 and references therein.

(9) Bailly, C.; Daumerie, M.; Legras, R.; Mercier, J. P. Makromol. Chem. 1986, 187, 1197.

(10) Brunelle, D. J.; Boden, E. P.; Shannon, T. G. J. Am. Chem. Soc. 1990, 112, 2399.

(11) Brunelle, D. J.; Shannon, T. G. Makromol. Chem., Macromol. Symp. 1991, 42/43, 155 .

(12) See, for example: Jones, R. O.; Gunnarsson, O. Rev. Mod. Phys. 1989, 61, 689; Density Functional Theory; NATO ASI Series, Series B, Physics; Vol. 337; Gross, E. K. U., Dreizler, R. M., Eds.; Plenum: New York, 1995.

(13) Montanari, B.; Ballone, P.; Jones, R. O. Macromolecules 1999, $32,3396$.

(14) Car, R.; Parrinello, M. Phys. Rev. Lett. 1985, 55, 2471.

(15) Montanari, B.; Ballone, P.; Jones, R. O. J. Chem. Phys. 1998, 108, 6947 and references therein.

(16) Perdew, J. P.; Burke, K.; Ernzerhof, M. Phys. Rev. Lett. 1996, 77, 3865 .

(17) Borrmann, A.; Jones, R. O. Chem. Phys. Lett. 1996, 252, 1.

(18) Baker, J.; Muir, M.; Andzelm, J. J. Chem. Phys. 1995, 102, 2063.

(19) Borrmann, A.; Montanari, B.; Jones, R. O. J. Chem. Phys. 1997, 106,8545

(20) Montanari, B.; Jones, R. O. Chem. Phys. Lett. 1997, 272, 347.

(21) Troullier, N.; Martins, J. M. Phys. Rev. B 1991, 43, 1993.

(22) Hutter, J.; et al. CPMD program version 3.0, Max-Planck-Institut für Festkörperforschung and IBM Research, 1990-99.

(23) For a discussion of electrostatic charges derived from quantum chemistry computations, see, for instance: Hawkins, G. D.; Cramer, C. J.; Truhlar, D. G. J. Chim. Phys. (Paris) 1997, 94, 1448.

(24) Mayer, I. Chem. Phys. Lett. 1983, 97, 270.

(25) Gerhards, M.; Kleinermanns, K. J. Chem. Phys. 1995, 103, 7392. Using the 6-31G(d,p) basis, Hartree-Fock and Møller-Plesset secondorder (MP2) calculations lead to $D_{\mathrm{e}}$ values of 7.26 and $6.99 \mathrm{kcal} / \mathrm{mol}$, respectively.

(26) Marchi, M.; Procacci, P. ORAC version 3.0; CECAM-ENS: Lyon, France. Parameters related to the $\mathrm{Li}$ atom were tuned using the results of the density functional calculations (bond lengths, binding energy). 